\title{
Recent Spin Structure Function Measurements from CLAS at Jefferson Laboratory
}

\author{
Robert Fersch* (for the CLAS Collaboration) \\ Christopher Newport University \\ E-mail: robert.fersch@cnu.edu
}

\begin{abstract}
The polarized electron accelerator at Jefferson Laboratory (JLab) in Newport News, Virginia, USA yields a robust program for the study of the spin physics of the nucleon. The CLAS detector and longitudinally polarized target in Hall-B at JLab were employed in multiple measurements of spin-structure functions for the proton and neutron in the resonance and DIS regions at beam energies of up to $6 \mathrm{GeV}$. Recently published results from the EG1 experiment present extensive measurements of the $g_{1}$ and $g_{2}$ structure functions for the proton over a wide kinematic range $\left(0.05<Q^{2}<5 \mathrm{GeV}^{2}\right.$ and $\left.1.08<W<3 \mathrm{GeV}\right)$. These data, together with data from the related EG4 and EG1-DVCS experiments in Hall-B, help constrain global models of structure functions, virtual photon asymmetries, and parton helicity distributions, and provide more precise values of higher-twist matrix elements in the framework of the Operator Product Expansion.
\end{abstract}

XXVI International Workshop on Deep-Inelastic Scattering and Related Subjects (DIS2018)

16-20 April 2018

Kobe, Japan

\footnotetext{
${ }^{*}$ Speaker.
} 


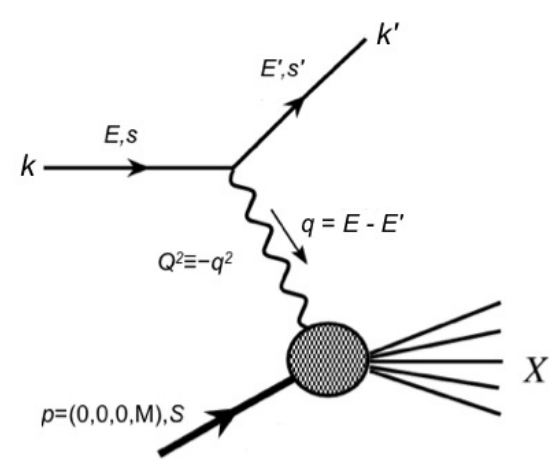

Figure 1: A schematic of inclusive electron-nucleon scattering. An incident electron (with 4-momentum $k$ and spin $s$ ) inelastically scatters via a virtual photon (with 4-momentum $q$ ) at polar angle $\theta$ from a nucleon (with 4-momentum $p$ and spin $S$ ) at rest in the lab frame with final 4-momentum $k^{\prime}$ and spin $s^{\prime} . E\left(E^{\prime}\right)$ represents the initial(final) energy of the scattered electron, and $X$ represents the final state hadron(s).

\section{Motivation and Formalism}

Understanding the structure of the nucleon in terms of its fundamental partonic (quark and gluon) degrees of freedom is a long-standing goal of hadronic physics. The partonic degrees of freedom (at leading twist) in a collinear momentum framework (where transverse momentum $k_{T}$ is assumed to be zero) can be described completely by three degrees of freedom per parton: $f(x)$ (the unpolarized distribution), $\Delta f(x)$ (helicity, or longitudinal spin distribution) and $\delta f(x)$ (transversity, or transverse spin distribution) [1]. In particular, quantifying the nucleon spin $J=\frac{1}{2}$ in terms of the sum of its quark and gluon helicities remains an open question since the discovery of the "Spin Puzzle" by the European Muon Collaboration [2]. Deep-inelastic electron scattering (DIS) from the nucleon (see Figure 1) provides a valuable method for constraining its partonic distributions.

In the Born approximation, the DIS cross-section for longitudinally-polarized electrons scattered from longitudinally-polarized nucleons can be described by two unpolarized structure functions $\left(F_{1}\right.$ and $\left.F_{2}\right)$ and two polarized structure functions $\left(g_{1}\right.$ and $\left.g_{2}\right)$ which depend solely on the 4-momentum transfer squared $Q^{2}=-q^{2}=4 E E^{\prime} \sin ^{2} \frac{\theta}{2}$ and virtual photon energy $v=E-E^{\prime}$. It is often conventional to express structure functions in terms of the Bjorken scaling variable

$$
x=\frac{Q^{2}}{2 p \cdot q}=\frac{Q^{2}}{2 M v}
$$

or the final-state invariant mass

$$
W=\sqrt{(p+q)^{2}}=\sqrt{M^{2}+2 M v-Q^{2}},
$$

in place of $v$, with the latter commonly used for data in the resonance region.

The polarized structure functions $g_{1}$ and $g_{2}$ carry fundamental information about the longitudinal spin distribution of the nucleon. The structure function $g_{1}$ can be interpreted in terms of the difference $\Delta q(x)=q \uparrow(x)-q \downarrow(x)$ of quark densities with helicity aligned( $\uparrow)$ vs. antialigned $(\downarrow)$ with the overall nucleon spin (for $j$ flavors) as

$$
g_{1}(x)=\frac{1}{2} \sum_{j} e_{j}^{2}\left[\Delta q_{j}(x)+\Delta \bar{q}_{j}(x)\right]
$$


where $e_{j}$ is the corresponding electric charge $(+2 / 3$ or $-1 / 3)$. The structure function $g_{2}$ is much more difficult to interpret in the quark-hadron picture; it contains non-suppressed higher-twist contributions, which therefore makes it a valuable experimental quality for studying polarized quarkgluon correlations, particularly "color polarizabilities" and forces in transversely polarized nucleons [3]. The reader is referred to Refs. [4, 5] for further information on these polarized structure functions and their theoretical interpretations.

\section{Experimental Overview and Analysis}

These proceedings focus on recently published results for $g_{1}$ polarized structure function for the proton from the EG1b experiment conducted in Hall-B at Jefferson Laboratory (JLab) during 2000-2001 [6]. Similar results for the deuteron (and extracted data for the neutron) can be found in Ref. [7].

The JLab electron accelerator produced continuous beams of electrons at energies up to $6 \mathrm{GeV}$ with longitudinal polarizations of approximately $70 \%$ measured via Møller polarimetry. The beam was varied between four (nominal) beam energies (1.6, 2.5, 4.2 and $5.7 \mathrm{GeV}$ ), and delivered to a fixed target in experimental Hall-B at a typical current of $20 \mathrm{nA}$. The longitudinally polarized target contained cylindrical cells filled with solid ${ }^{15} \mathrm{NH}_{3}$ and ${ }^{15} \mathrm{ND}_{3}$ beads immersed in liquid helium (LHe), typically maintained at $75 \%$ and $30 \%$ polarizations, respectively, by dynamic nuclear polarization (DNP) for both proton and deuteron scattering. Separate ${ }^{12} \mathrm{C}$ and empty (LHe only) cells were employed for unpolarized background subtraction [8].

The CEBAF Large Acceptance Spectrometer (CLAS) in Hall-B [9] provided full azimuthal detection of all (inclusive) scattered electrons between polar angles as low as $8^{\circ}$ and as high as $48^{\circ}$. Incident scattered electrons were counted (normalized by total beam charge) in bins of $W$ and $Q^{2}$ for the cases where the electron beam and target polarizations were aligned $\left(n^{+}\right)$and antialigned $\left(n^{-}\right)$. A normalized asymmetry was calculated as

$$
A_{\|} \approx \frac{1}{P_{b} P_{t} F_{D F}} \frac{n^{+}-n^{-}}{n^{+}+n^{-}} .
$$

Here, $F_{D F}$ is a "dilution factor" accounting for unpolarized ${ }^{15} \mathrm{~N}$, LHe, and target cell wall backgrounds, calculated using Born cross-section ratios from an (unpolarized) model incorporating radiative corrections, along with data from ${ }^{12} \mathrm{C}$ and empty target cell runs (to constrain effective thicknesses of all target components). $P_{b} P_{t}$ is the product of beam and target polarizations, determined by normalization of $A_{\|}$, as measured at the elastic scattering peak at $W=0.938 \mathrm{GeV}$, to the expected elastic asymmetry, which was precisely determined as a function of the known electromagnetic form factors $G_{E}\left(Q^{2}\right)$ and $G_{M}\left(Q^{2}\right)$ [10]. Additional corrections (not shown in Eq. 2.1) and systematic uncertainties were implemented for $e^{+} e^{-}$contamination, $\pi^{-}$contamination, and residual ${ }^{15} \mathrm{~N}$ polarization. Finally, radiative corrections (both internal and external) were made using a method similar to those used in earlier measurements of $A_{\|}$at SLAC [11]. Details on the bulk of this analysis can be found in Ref. [6].

The structure function $g_{1}$ could then be determined from $A_{\|}$as

$$
g_{1}=\frac{\tau}{1+\tau}\left(\frac{A_{\|}}{D}+(\gamma-\eta) A_{2}\right) F_{1}
$$


where $\tau, \eta$ and $\gamma$ are kinematic functions of $E, Q^{2}$ and $W$. The structure function $F_{1}$ was obtained from a model fit to (unpolarized) world data. The (considerably less well-constrained) virtual photon asymmetry $A_{2}$ was also modeled, but it makes only a small contribution to $g_{1}$. The "depolarization factor" $D$ is given by

$$
D=\frac{1-E^{\prime} \varepsilon / E}{1+\varepsilon R}
$$

where $\varepsilon$ is another kinematic factor and

$$
R=\frac{F_{2}\left(1+\frac{1}{\tau}\right)}{2 x F_{1}}+1
$$

is a function of the $F_{1}$ and $F_{2}$ structure functions, again modeled from world data.

Results for the $g_{2}$ structure function have also been published. Though of value as a global constraint on $g_{2}$ due to the scarcity of world data on this proton structure function, these data are of poor precision compared to those available from this analysis for $g_{1}$. Also available are related results for the asymmetries $A_{1}$ and $A_{2}$ for the virtual photon in Fig. 1, with precisions corresponding (approximately) to those of $g_{1}$ and $g_{2}$, respectively. These asymmetries are useful for constraining quark distribution functions and pQCD models, particularly in the high- $x$ region (e.g. $[12,13])$. Also published are measurements of various moments of the $g_{1}$ structure function, e.g.

$$
\Gamma_{1}\left(Q^{2}\right) \equiv \int_{0}^{1} g_{1}\left(x, Q^{2}\right) d x
$$

which are useful for investigating the QCD-structure of the nucleon, in part for their connection to various "sum rules" governing the behavior of polarized structure functions (e.g. [14]). The reader is hereby again referred to Ref. [6] for these results and a more detailed explanation.

\section{Results for the $g_{1}$ Structure Function}

These published results contain the most comprehensive set of data for the $g_{1}$ structure function for the proton available in the resonance region, extending into the near DIS kinematic region. These results, and how they segue into the context of DIS, are summarized in Fig. 2. One can see how a dramatic dependence on $Q^{2}$ at low $Q^{2}$ in the lower $x$ bins (where the resonance region dominates) makes way to a smooth approach in the DIS limit. Variations in this upper limit can serve as a study of scaling violations due to $\mathrm{pQCD}$ evolution.

The precision of these data in the resonance region also provides the best opportunity yet available for the study of quark-hadron duality for the $g_{1}$ structure function. In studies of quark-hadron (i.e. Bloom-Gilman) duality, nucleon structure functions (and other observables) are compared in terms of hadronic and partonic degrees of freedom [16]. In short, extrapolated (next-to-leading order, or NLO) fits of structure functions in the DIS region at high $Q^{2}$ are compared to the results in the resonance region, averaged over $x$ :

$$
\left\langle g_{1}\left(Q^{2}\right)\right\rangle=\frac{\int_{x_{l}}^{x_{h}} g_{1}\left(x, Q^{2}\right) d x}{x_{h}-x_{l}} .
$$

A comparison of $\left\langle g_{1}\left(Q^{2}\right)\right\rangle$ (where resonance structure due to hadronic degrees of freedom dominates) to the NLO DIS fit of $g_{1}$ (for which partonic degrees of freedom dominate) provides a test of 


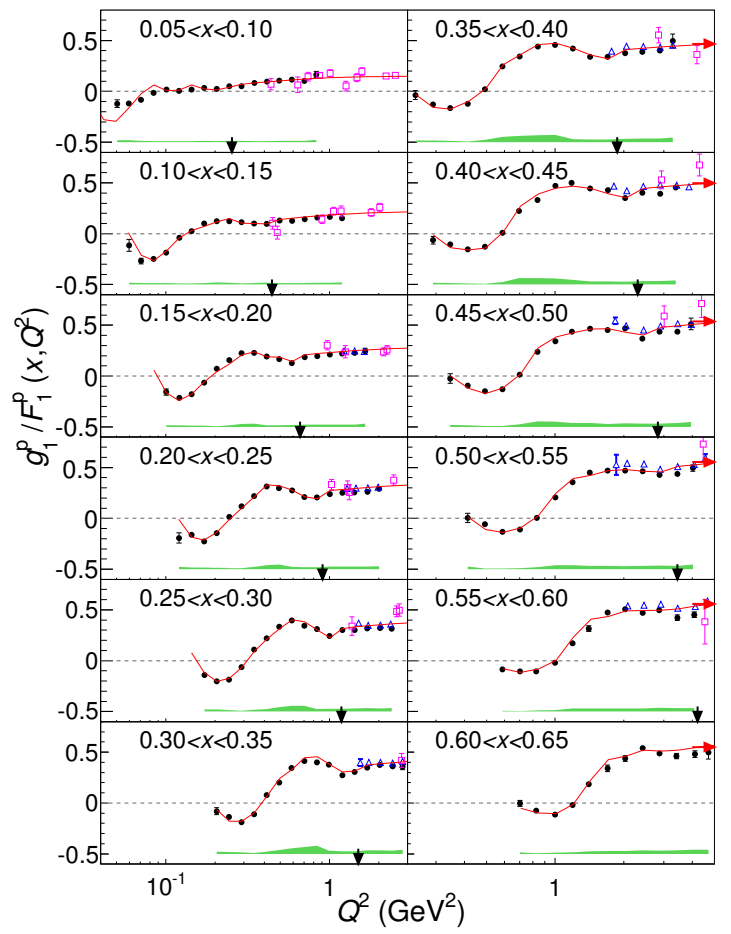

Figure 2: Plots of $g_{1} / F_{1}$ for the proton versus $Q^{2}$ for different $x$-ranges of the EG1b data. Blue triangles and magenta squares correspond to EG1-DVCS ([15]) and SLAC E143 ([11]) data, respectively. The downwardpointing black arrows indicate the edge of the resonance region at $W=2 \mathrm{GeV}$, while the horizontal red arrows indicate the expected DIS limit of world data for $g_{1} / F_{1}$. From Ref. [6].

the equivalence of these two pictures of nucleon structure [17]. Tests of quark-hadron duality are possible for the whole resonance region (i.e. "global duality") or for localized resonance regions (i.e. "local duality"). Results for the test of global duality for the EG1 proton data are shown in Fig. 3.

The conclusion of inclusive polarized electron-proton scattering analysis in the CLAS EG1b experiment provides a wealth of new data for polarized structure functions of the nucleon. In conjunction with other experiments in CLAS, such as the similar EG4 experiment, which extends these measurements to a lower $Q^{2}$ range [18], and the EG1-DVCS experiment, which extends these measurements to a higher $Q^{2}$ range [15], a richer picture of the nucleon emerges. Together with other JLab data, substantial impact has been made to the understanding of polarized parton distribution functions (PDFs) through a global analysis of data [19], which will continue into the future with the recent upgrade of the JLab electron accelerator to $12 \mathrm{GeV}$ beam energies.

\section{Acknowledgements}

We would like to acknowledge the outstanding efforts of the staff of the Accelerator and the Physics Divisions at Jefferson Lab that made this experiment possible. This work was supported in part by the U.S. Department of Energy and the National Science Foundation, the Italian Istituto Nazionale di Fisica Nucleare, the French Centre National de la Recherche Scientifique, the French 


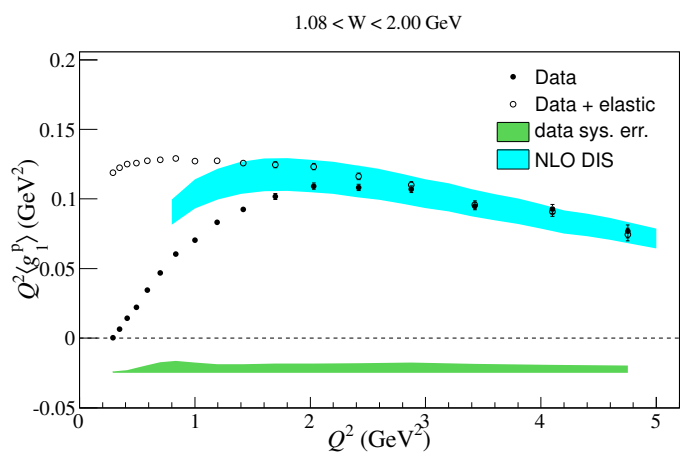

Figure 3: The $Q^{2}$ dependence of $Q^{2}\left\langle g_{1}\right\rangle$ for the proton averaged over the resonance region in $x$, compared to its corresponding extrapolated NLO DIS fit (cyan band). The open circles represent the same data with the contribution from $e p$ elastic scattering added in. See the text for details. From Ref. [6].

Commissariat à l'Energie Atomique, the Emmy Noether grant from the Deutsche Forschungs Gemeinschaft, the United Kingdom's Science and Technology Facilities Council, and the National Research Foundation of Korea. The Jefferson Science Associates (JSA) operates the Thomas Jefferson National Accelerator Facility for the United States Department of Energy under Contract No. DE-AC05-06OR23177.

\section{References}

[1] B. Lampe and E. Reya, Phys. Rept. 332, 1-163 (2000).

[2] J. Ashman, et al., Phys. Lett. B206, 364 (1988).

[3] M. Burkardt, AIP Conf. Proc. 1155, 26 (2009).

[4] S. Kuhn, J. P. Chen and E. Leader, Prog. Part. Nucl. Phys. 63, 1 (2009).

[5] C. A. Aidala, S. D. Bass, D. Hasch and G. K. Mallot, Rev. Mod. Phys. 85, 655 (2013).

[6] R. Fersch, N. Guler, et al., Phys. Rev. C96 065208 (2017)

[7] N. Guler, R. Fersch, et al., Phys. Rev. C92 055201 (2015)

[8] C. Keith et. al., Nucl. Instrum. Meth. A501, 327 (2003).

[9] B. Mecking et. al., Nucl. Instrum. Meth. A503, 513 (2003).

[10] J. Arrington, Phys. Rev. C69, 022201 (2004).

[11] K. Abe et. al., Phys. Rev. Lett. 79, 26 (1997).

[12] N. Isgur, Phys. Rev. D59, 034013 (1999).

[13] H. Avakian, S. J. Brodsky, A. Deur and F. Yuan, Phys. Rev. Lett. 99, 082001 (2007).

[14] E. V. Shuryak and A. I. Vainshtein, Nucl. Phys. B201, 141 (1982).

[15] Y. Prok, et al., Phys. Rev. C90, 025212 (2014).

[16] W. Melnitchouk, R. Ent, and C. Keppel, Phys. Rept. 406, 127 (2005).

[17] P. E. Bosted, et al., Phys. Rev. C75, 035203 (2007).

[18] K. Adhikari, et al., Phys. Rev. Lett. 120, 062501 (2018).

[19] W. Meltinchouk, et al., Phys. Rev. D93, 074005 (2016). 